\title{
Atopic dermatitis-like skin lesions with IgE hyperproduction and pruritus in KFRS4/Kyo rats.
}

\section{$\operatorname{AUTHOR}(\mathrm{S}):$}

Kuramoto, Takashi; Yokoe, Mayuko; Tanaka, Daisuke; Yuri, Azusa; Nishitani, Ai; Higuchi, Yuki; Yoshimi, Kazuto; ... Hiai, Hiroshi; Kabashima, Kenji; Serikawa, Tadao

\section{CITATION:}

Kuramoto, Takashi ...[et al]. Atopic dermatitis-like skin lesions with lgE hyperproduction and pruritus in KFRS4/Kyo rats.. Journal of dermatological science 2015, 80(2): 116-123

\section{ISSUE DATE:}

2015-11

URL:

http://hdl.handle.net/2433/207421

\section{RIGHT:}

(c) 2015. This manuscript version is made available under the CC-BY-NC-ND 4.0 license

http://creativecommons.org/licenses/by-nc-nd/4.0/; The full-text file will be made open to the public on 1 December 2016 in accordance with publisher's 'Terms and Conditions for Self-Archiving'.; This is not the published version. Please cite only the published version,; この論文は出版社版でありません。引用の際には出版社版をご確認ご利用ください。 


\section{Atopic dermatitis-like skin lesions with IgE hyperproduction and pruritus in KFRS4/Kyo rats}

Takashi Kuramoto ${ }^{a}{ }^{*}$ Ph.D., Mayuko Yokoe $^{\mathrm{a}}$, Daisuke Tanaka ${ }^{\mathrm{a}}$, Azusa Yuri ${ }^{\mathrm{a}}$, Ai Nishitani $^{\mathrm{a}}$, Yuki Higuchi ${ }^{\mathrm{a}}$, Kazuto Yoshimi ${ }^{\mathrm{a}, 1}$ Ph.D., Miyuu Tanaka ${ }^{\mathrm{a}, \mathrm{b}}$ DVM Ph.D., Mitsuru Kuwamura ${ }^{\mathrm{b}}$ DVM Ph.D., Hiroshi Hiai ${ }^{\mathrm{c}}$ MD Ph.D., Kenji Kabashima ${ }^{\mathrm{d}}$ MD Ph.D., Tadao Serikawa ${ }^{\mathrm{a}, \mathrm{e}}$ DVM Ph.D.

${ }^{a}$ Institute of Laboratory Animals, Graduate School of Medicine, Kyoto University, Yoshida-konoe-cho, Sakyo-ku, Kyoto 606-8501, Japan

baboratory of Veterinary Pathology, Osaka Prefecture University, Izumisano, Osaka, 598-8531, Japan

${ }^{\mathrm{c}}$ Medical Innovation Center, Graduate School of Medicine, Kyoto University, Shogoin-kawara-cho, Sakyo-ku, Kyoto 606-8507, Japan

${ }^{\mathrm{d}}$ Department of Dermatology, Graduate School of Medicine, Kyoto University, Shogoin-kawara-cho, Sakyo-ku, Kyoto 606-8507, Japan

eLaboratory of Pharmacology, Osaka University of Pharmaceutical Sciences, Takatsuki, Osaka 569-1094, Japan

${ }^{1}$ Present address: Mouse Genomics Resource Laboratory, National Institute of Genetics, Mishima, Shizuoka 411-8540, Japan 
*Correspondence should be addressed to: Takashi Kuramoto, Ph.D.

Institute of Laboratory Animals, Graduate School of Medicine, Kyoto University, Yoshidakonoe-cho, Sakyo-ku, Kyoto 606-8501, Japan

Tel: +81-75-753-4494

Fax: +81-75-753-4409

E-mail: tkuramot@anim.med.kyoto-u.ac.jp

Funding: This work was supported in part by the Japan Society for the Promotion of Science program (KAKENHI 26290031) and the KAC 35th anniversary grant.

Conflicts of interest: The authors have no conflict of interest to declare

Running title: Novel rat model of atopic dermatitis

Text word count: 4,368

Number of references: 31

Number of Tables: 0

Number of Figures: 4 


\section{Abstract}

Background: Rats showing spontaneous atopic dermatitis (AD)-like skin lesions were observed in the Kyoto fancy rat stock 4 (KFRS4) strain breeding colony.

Objective: To establish the KFRS4 rat as a model of AD.

Methods: The clinical symptoms of AD-like skin lesions were assessed by scoring the degree of dermatitis and examining scratching behavior. The transepidermal water loss was measured to evaluate skin barrier function. Cells infiltrating the skin lesions were identified using histological and immunohistological analyses. IgE and cytokine levels were measured to examine immune status. An ointment treatment experiment was carried out to characterize dermatitis in the KFRS4 rats.

Results: Dermatitis initially appeared around 4 months of age and rapidly worsened from 6 to 8 months of age. The skin lesions accompanied scratching behavior and were predominantly observed in females. The increased transepidermal water loss indicated skin barrier dysfunction. Extensive infiltration of eosinophils, mast cells and lymphocytes was observed in the skin lesions. The plasma IgE level increased in accord with increasing severity of dermatitis. The Th2 and Th17 cytokine mRNA levels were significantly higher in the skin-draining lymph nodes than those in the 
non-skin-draining lymph nodes. It was demonstrated that betamethasone improved the symptoms of dermatitis. These findings demonstrated that dermatitis in the KFRS4 rats closely resembled that seen in human AD.

Conclusion: Female KFRS4 rats have the potential to serve as an animal model of human AD.

Keywords: Atopic dermatitis, Betamethasone, IgE, Pruritus, Rats 


\section{Abbreviations}

$\mathrm{AD}$, atopic dermatitis

HE, hematoxylin-eosin

KFRS4, Kyoto fancy rat stock 4

LN, lymph node

TB, toluidine blue

TEWL, transepidermal water loss

TSLSS, total skin lesion severity score 


\section{Introduction}

Atopic dermatitis (AD) is a relapsing chronic inflammatory skin disease characterized by eczematous skin lesions and intense pruritus [1]. AD affects at least $15 \%$ of children and $2-10 \%$ of adults in industrialized countries, and there is growing evidence of an increase in prevalence [2]. AD is often associated with elevated serum immunoglobulin E (IgE) levels and a personal or family history of type I allergies, allergic rhinitis and asthma.

The etiology of AD is highly complex, but several lines of evidence suggest that a genetic predisposition leads to a defective skin barrier and a dysregulated immune response [3, 4]. Environmental triggers such as food allergens, inhalable respiratory allergens, irritating substances and infectious microorganisms [5, 6] play an important role in the development of AD. Scratching induced by pruritus disrupts skin barrier functions, which also contributes to the development of $\mathrm{AD}$ [7]. Thus, skin barrier function, immunology, pruritus and the environment, as well as interactions among these factors, are important considerations when examining the mechanisms underlying the development of AD.

To understand the complex pathogenesis of $\mathrm{AD}$ in the development of effective 
procedures for diagnosis, prevention and treatment, animal models of $\mathrm{AD}$ are essential. These models allow the in-depth investigation of pathogenesis and provide invaluable tools for diagnostic and pharmaceutical purposes. There are various mouse models of AD available, including models induced by epicutaneous sensitization, genetically engineered models and spontaneous models [8]. Among them, NC/Nga mice have been a widely used AD-like dermatitis model with an elevation in IgE levels and intense pruritus; this is primarily influenced by mite infection in specific pathogen free (SPF) conditions $[9,10]$. Although mouse models are primarily used, rat models of AD are also expected to be developed because rats are widely used in biomedical and pharmaceutical research areas.

Kyoto Fancy Rat Stock 4 (KFRS4) rats have been developed from hybrids between the fancy rat and PVG/Seac inbred strain rats [11]. During inbreeding, several mutant phenotypes carried by the fancy rat are selected. As such, KFRS4 rats carry mutations that include bright coat color, hooded coat pattern, white spotting on the head and cataracts [12-15].

In the present study, we newly observed spontaneous dermatitis that accompanied scratching behavior in KFRS4 rats. This dermatitis appeared around 6 
months of age and worsened progressively during the next 2 months. It seemed that there was a sex difference in incidence; almost all female rats but only some males were affected. To characterize KFRS4 rats as a model of AD, we examined the clinical symptoms, scratching behavior, histological analysis of skin lesions and immune status. In addition, we performed a treatment experiment regarding dermatitis on the KFRS4 rats using the corticosteroid betamethasone. 


\section{Materials and Methods}

\subsection{Rats}

KFRS4/Kyo (NBRP\#0572) and PVG/Seac (NBRP\#0080) rat strains were sourced from the National BioResource Project-Rat, Kyoto University (Kyoto, Japan). Rats were bred in conventional conditions in which they were free of mites but were infected by pinworms (Syphacia muris). All animal experiments were approved by the Animal Research Committee of Kyoto University and were conducted according to the Regulations on Animal Experimentation of Kyoto University.

\subsection{Reagents}

Mouse anti-rat CD4 (clone, W3/25; 1:5000) and mouse anti-rat CD8 alpha (clone, OX-8; 1:500) were purchased from AbD Serotec (Raleigh, North Carolina, USA). Heparinoid cream (Hirudoid ${ }^{\circledR} \quad$ Soft) and betamethasone dipropionate (Rinderon ${ }^{\circledR}-\mathrm{V}$ Ointment $0.12 \%$ ) were purchased from Maruho Co., Ltd. (Osaka, Japan) and Shionogi \& Co., Ltd. (Osaka Japan), respectively. 


\subsection{Clinical observation}

The clinical severity of the skin lesions was scored in male $(n=8)$ and female $(n=8)$ KFRS4 rats according to the macroscopic diagnostic criteria, termed the total skin lesion severity score (TSLSS) [16]. Briefly, the TSLSS was designated as the sum of the individual scores, graded as 0 (none), 1 (mild), 2 (moderate) and 3 (severe) for the symptoms of erythema, eczema, crust, edema and erosion in six regions of the body, namely the face, head and neck, forelegs, hind legs, dorsal and ventral regions of the trunk.

\subsection{Histology}

For histological analysis, major organs including the skin were harvested, fixed in buffered $10 \%$ formalin, embedded in paraffin and stained with hematoxylin-eosin (HE) [17]. For examination of mast cells, skin sections were stained with toluidine blue (TB). The number of mast cells was calculated as the average from 20 fields of dermatitis ( $(=4)$ and normal $(n=4)$ skin samples (original magnification, $\times 400)$. For immunohistochemistry, $10-\mu \mathrm{m}$ frozen sections of the skin from 6-month-old KFRS4 rats were fixed in methanol:acetone (1:1) for $10 \mathrm{~min}$ at $4^{\circ} \mathrm{C}$ and washed in PBS. 
Sections were treated with $10 \%$ goat serum in PBS and then incubated for $1 \mathrm{~h}$ at room temperature with anti-CD4 and CD8 antibodies. After incubation with the primary antibodies, sections were treated with peroxidase-conjugated secondary antibody (Histofine Simplestain MAX-PO: Nichirei, Tokyo, Japan) and signals were visualized using a DAB substrate kit (Nichirei).

\subsection{Measurement of plasma IgE}

Rats were anesthetized with isoflurane and blood samples were collected from the tail tip using heparinized hematocrit tubes. Total plasma IgE levels were measured using a rat IgE EIA kit (Takara Bio Inc., Otsu, Shiga, Japan).

\subsection{Skin barrier function}

The dorsal region of the skin was shaved in female KFRS4 $(n=6)$ and PVG (n =6) rats before measurement. To evaluate the inside-to-outside barrier function, transepidermal water loss (TEWL) was measured using a Tewameter Vapo Scan (Asahi Biomed, Tokyo, Japan) at $24^{\circ} \mathrm{C}$ and $46 \%$ relative humidity at 5 and 9 weeks of age. TEWL measurements were carried out in triplicate for each rat. 


\subsection{Scratching behavior}

Pruritus was evaluated in terms of the level of scratching behavior. Rats aged 4 months $(n=6), 6$ months $(n=6)$ and 8 months $(n=6)$ were placed individually in plastic cages $(30 \mathrm{~cm} \times 30 \mathrm{~cm} \times 20 \mathrm{~cm})$. After an acclimation period $(60 \mathrm{~min})$, the number of scratching movements was counted in the morning during the 60 -min observation period.

\subsection{Treatment of dermatitis}

Four-month-old female KFRS4 rats $(\mathrm{n}=18)$ were divided into three groups. Group 1 rats were untreated $(n=6)$, group 2 rats were treated with heparinoid cream $(n$ $=6)$ and group 3 rats were treated with betamethasone $(n=6)$. Ointment was applied to the skin of the head and neck three times per week. Every 4 weeks, the clinical observation of the skin, an examination of scratching behavior and measurement of plasma IgE were performed. At 7 months of age, animals were sacrificed under isoflurane anesthesia and the spleen, adrenal glands, lymph nodes (LNs) and skin were harvested for histological analysis. 


\subsection{Quantitative real-time polymerase chain reaction}

Thirteen skin-draining LNs and 8 non skin-draining LNs (mesenteric and renal) were harvested from 3 KFRS4 rats. Messenger RNA (mRNA) was isolated using ISOGEN II (Nippon Gene Co., Ltd, Tokyo, Japan) according to the manufacturer's instructions. Real-time polymerase chain reaction was performed using the Thermal Cycler Dice Real Time System (Takara Bio Inc., Otsu, Shiga, Japan) with SYBR Premix Ex Taq II (Takara Bio Inc.). By monitoring the amplification curves of a test sample and reference samples that contained $10^{1}$ to $10^{6}$ molecules of the gene of interest, the number of target molecules in the test sample was analyzed. The number of target molecules was normalized to that of peptidylprolyl isomerase A (Ppia) as an internal control [18]. The primers used are listed in Supplementary Table 1.

\subsection{Hinkelmann staining}

Peripheral blood samples were collected from the tail veins of dermatitis-affected (6-8 months of age; $\mathrm{n}=7$ ) and non-affected (4-5 months of age; $\mathrm{n}=$ 6) female KFRS4 rats. Blood samples (10 $\mu \mathrm{L})$ were stained with Hinkelmann's solution (Muto Pure Chemicals, Tokyo, Japan). 


\subsection{Statistical analysis}

Data were analyzed using an unpaired two-tailed $t$-test. $P$ values $<0.05$ were considered significant. All results are shown as the mean \pm standard deviation (SD). 


\section{Results}

\subsection{Spontaneous dermatitis in KFRS4 rats}

Skin lesions initially appeared as erythema on the lips or forelegs at around 4 months of age. Until 6 months of age, mild skin lesions consisting of erythema, eczema, and crust were seen on the skin of the KFRS4 rats. With increasing age, severe edema and erosion were seen, particularly in the skin of the head and neck region and the dorsal region of the trunk (Fig. 1A). These skin lesions were observed in $100 \%$ of 8-month-old female and 50\% of 8-month-old male KFRS4 rats examined. TSLSS was significantly higher in females than in the age-matched males (Fig. 1B). The frequency of scratching behavior during the 60-min observation period dramatically increased after 6 months of age in female KFRS4 rats (Fig. 1C). In contrast, no skin lesions or scratching behavior were observed in PVG rats, a parental strain of KFRS4.

\subsection{Skin barrier function in KFRS4 rats}

Because skin barrier dysfunction is a common characteristic of $\mathrm{AD}[7,19]$, we measured the TEWL, an established indicator of barrier function. The TEWL of KFRS4 
rats was significantly higher than that of PVG rats both at 5 and 9 weeks of age (Fig. 1D) when no dermatitis was observed clinically and histologically. This finding indicates a defect in the skin barrier function of KFRS4 rats before the appearance of dermatitis.

\subsection{Histological examination of skin lesions in female KFRS4 rats}

The histology of early skin lesions in KFRS4 rats showed epidermal hyperplasia with mild spongiosis, parakeratosis, and infiltration of lymphocytes in the epidermis and superficial dermis (Fig. 2A). As the lesions progressed, there was extensive infiltration of eosinophils and lymphocytes in the dermis. A skin ulcer that was covered by crusts formed by fibrin exudate and necrotic tissue debris was often observed (Fig. 2B). In addition, mast cells that were detected using TB staining increased in number in the dermis of dermatitis-affected skin as compared with non-dermatitis skin (Fig. 2C). The infiltration of both $\mathrm{CD}^{+}$and $\mathrm{CD}^{+} \mathrm{T}$ cells was observed in the dermatitis-affected skin (Fig. 2D). The number of $\mathrm{CD}^{+}$cells was significantly increased in the dermatitis region as compared with the non-dermatitis region (78.3 \pm 15.6 vs $0.33 \pm 0.50 ; P<0.01)$. In addition, the number of $\mathrm{CD}^{+}$cells was 
significantly increased $(18.2 \pm 4.68$ vs $0.50 \pm 0.53$; $\mathrm{P}<0.01$; Fig. $2 \mathrm{D})$. None of these changes were observed in PVG rats.

\subsection{Immune status of KFRS4 rats in the steady state}

To elucidate the immune status of female KFRS4 rats, we measured the levels of total IgE, because the increased severity of $\mathrm{AD}$ is reported to be correlated with elevated IgE levels [16, 20]. IgE levels were significantly higher in female KFRS4 rats than in age-matched male KFRS4 rats from the onset (5 months of age) to the exacerbation phase of dermatitis (7 months of age) (Fig. 3A). To examine the immune status of KFRS4 rats showing severe dermatitis, we measured the Th1 (interferon (IFN)- $\gamma$ ), Th2 (interleukin (IL)-4), Th17 (IL-17A, IL-22 and IL-23), and Treg (IL-10), plasma cell (tumor necrosis factor [TNF] $\alpha$ ) cytokine mRNA levels of the skin-draining mandibular and axillary LNs of the affected rats, and compared them with those of the non-skin-draining LNs. The mRNA levels of IL-22, IL-23, IL-4 and TNF $\alpha$ were significantly higher in the skin-draining LNs than in the non-skin-draining LNs (Fig. 3B). Furthermore, we examined the number and proportion of eosinophils in the peripheral blood of KFRS4 rats; this was because an increase in the number of 
eosinophils has been found in most patients with $\mathrm{AD}$ and correlates with disease activity [21]. Eosinophils increased significantly in number in dermatitis-affected KFRS4 rats as compared with non-affected rats (Fig. 3C). The proportion of eosinophils in leukocytes was also significantly increased $(2.7 \pm 1.1 \%$ in non-affected rats vs $12.4 \pm 4.0 \%$ in dermatitis-affected rats; $P<0.01)$.

These findings suggest that Th2, Th17 cells and eosinophils, similar to those in human $\mathrm{AD}$ patients [22], play an important role in the pathogenesis of dermatitis in KFRS4 rats.

\subsection{Suppression of dermatitis by steroids}

Because topical steroid treatment is commonly used for AD, we carried out ointment treatment experiments in female KFRS4 rats. Heparinoid cream was used as a control. When compared with untreated and heparinoid-treated rats, the rats treated with betamethasone ointment showed a significant improvement in TSLSS (Fig. 4A) and scratching behavior (Fig. 4B). The IgE level in betamethasone-treated rats tended to be lower than in non-treated or heparinoid-treated rats, but was not significantly different among them. 
Histological examination showed that marked skin inflammation and ulceration were evident in the non-treated or heparinoid cream-treated rats but were virtually absent in the betamethasone-treated rats (Fig. 4C). These findings indicate that betamethasone is effective in treating dermatitis in KFRS4 rats, which is also observed in patients with $\mathrm{AD}$. 


\section{Discussion}

KFRS4 rats showed spontaneous dermatitis that accompanied scratching behavior, elevation of plasma IgE, and Th2 and Th17 cytokine mRNAs. The histology of the skin lesion was characterized by the infiltration of lymphocytes, eosinophils and mast cells. The increased TEWL indicated skin barrier dysfunction in KFRS4 rats. In addition, the treatment with betamethasone ointment had marked effects on suppression of dermatitis. These clinical, immunological and histological features of KFRS4 rat dermatitis closely resemble those seen in human AD. Thus, KFRS4 rats are considered to be a good model of AD.

The microbiological environments where animals are bred play important roles in the development of dermatitis. NC/Nga mice develop dermatitis when bred in conventional conditions or under specific pathogen free conditions with an infection of house dust mites [9]. Staphylococcus aureus colonization on the skin drives inflammation in spontaneous or genetically modified rodent models of $\mathrm{AD}[23,24]$. KFRS4 rats are bred without mites but with infection by pinworms (Syphacia muris), which are known to influence allergic reactions in mice [25]. Thus, pinworms may be involved in the development of dermatitis in KFRS4 rats through modulation of the 
immune system or the generation of allergens. The development of pinworm-free KFRS4 rats in specific pathogen free conditions would be required to address the microbiological environment issue.

Although no sex differences have been observed in human AD patients [26, 27] or mouse models of $\operatorname{AD}[9,16,28]$, we found a clear sex difference in the dermatitis of KFRS4 rats. Female KFRS4 rats developed much more severe dermatitis as compared with age-matched males. Because male and female KFRS4 rats are kept in identical environments, female sex apparently predisposes KFRS4 rats to AD-like skin lesions. There are sex differences in immune responses, especially from the perspective of collagen vascular diseases, and the immune response plays a pivotal role in the development of $\operatorname{AD}[7,29,30]$. Thus, it is possible to consider that the sex difference may influence the development of $\mathrm{AD}$ in certain conditions. The sex difference in the immune response is attributed not only to differences in sex hormones, such as estrogen, testosterone and progesterone, but also to X chromosome gene contributions [30]. The $\mathrm{X}$ chromosome gene contributions would appear as $\mathrm{X}$-linked diseases in which males show the severe phenotype and females are relatively unaffected. Thus, it is likely that sex hormones may contribute to the higher incidence of dermatitis found in female 
KFRS4 rats. To this end, developing ovariectomy or orchiectomy in KFRS4 rats would be needed.

We carried out an ointment treatment experiment in KFRS4 rats and found that steroid (betamethasone) was effective in suppressing dermatitis. This finding suggested that KFRS4 rats are suitable for the evaluation of new drugs or treatments for AD. Owing to $100 \%$ prevalence of dermatitis, female KFRS4 rats could be considered as providing an efficient bioassay system. Indeed, in KFRS4 rats, skin barrier dysfunction occurred before the appearance of dermatitis. Daily application of moisturizer during the neonatal period reduces the risk of $\mathrm{AD}$ [31]. Thus, it would be interesting to examine the preventive effect of moisturizer on dermatitis in KFRS4 rats before the appearance of dermatitis.

In summary, KFRS4 rats spontaneously developed dermatitis that accompanied an elevation of IgE and scratching behavior. The dermatitis was evidently suppressed by topical application of betamethasone. In addition, increased TEWL was observed before the onset of dermatitis, which indicates that there was skin barrier dysfunction in KFRS4 rats. Taken together, KFRS4 rats have the potential to serve as an animal model of human AD that would be useful for diagnostic and pharmaceutical purposes, and 
could provide insights into the possible mechanisms associated with human AD. 


\section{Acknowledgements}

This work was supported in part by the Japan Society for the Promotion of Science program (KAKENHI 26290031) and the KAC 35th anniversary grant. We are thankful to the National BioResource Project-Rat (http://www.anim.med.kyoto-u.ac.jp/NBR/) for providing the rat strains (KFRS4/Kyo and PVG/Seac). 


\section{References}

[1] T. Bieber, Atopic dermatitis, N Engl J Med 358 (2008) 1483-1494.

[2] C.R. Totri, L. Diaz, L.F. Eichenfield, 2014 update on atopic dermatitis in children, Curr Opin Pediatr 26 (2014) 466-471.

[3] C.N. Palmer, A.D. Irvine, A. Terron-Kwiatkowski, Y. Zhao, H. Liao, S.P. Lee, et al., Common loss-of-function variants of the epidermal barrier protein filaggrin are a major predisposing factor for atopic dermatitis, Nat Genet 38 (2006) 441-446.

[4] I.H. Kuo, T. Yoshida, A. De Benedetto, L.A. Beck, The cutaneous innate immune response in patients with atopic dermatitis, J Allergy Clin Immunol 131 (2013) 266-278.

[5] M.M. Bergmann, J.C. Caubet, M. Boguniewicz, P.A. Eigenmann, Evaluation of food allergy in patients with atopic dermatitis, J Allergy Clin Immunol Pract 1 (2013) $22-28$.

[6] T. Werfel, A. Kapp, Environmental and other major provocation factors in atopic dermatitis, Allergy 53 (1998) 731-739. 
[7] K. Kabashima, New concept of the pathogenesis of atopic dermatitis: interplay among the barrier, allergy, and pruritus as a trinity, J Dermatol Sci 70 (2013) 3-11.

[8] H. Jin, R. He, M. Oyoshi, R.S. Geha, Animal models of atopic dermatitis, J Invest Dermatol 129 (2009) 31-40.

[9] H. Matsuda, N. Watanabe, G.P. Geba, J. Sperl, M. Tsudzuki, J. Hiroi, et al., Development of atopic dermatitis-like skin lesion with IgE hyperproduction in NC/Nga mice, Int Immunol 9 (1997) 461-466.

[10] T. Sasakawa, Y. Higashi, S. Sakuma, Y. Hirayama, Y. Sasakawa, Y. Ohkubo, et al., Atopic dermatitis-like skin lesions induced by topical application of mite antigens in NC/Nga mice, Int Arch Allergy Immunol 126 (2001) 239-247.

[11] T. Kuramoto, M. Yokoe, K. Yagasaki, T. Kawaguchi, K. Kumafuji, T. Serikawa, Genetic analyses of fancy rat-derived mutations, Exp Anim 59 (2010) 147-155.

[12] L.A. Quina, T. Kuramoto, D.V. Luquetti, T.C. Cox, T. Serikawa, E.E. Turner, Deletion of a conserved regulatory element required for Hmx1 expression in craniofacial mesenchyme in the dumbo rat: a newly identified cause of congenital ear malformation, Dis Model Mech 5 (2012) 812-822. 
[13] T. Kuramoto, S. Nakanishi, M. Ochiai, H. Nakagama, B. Voigt, T. Serikawa, Origins of albino and hooded rats: implications from molecular genetic analysis across modern laboratory rat strains, PLoS One 7 (2012) e43059.

[14] K. Watanabe, K. Wada, T. Ohashi, S. Okubo, K. Takekuma, R. Hashizume, et al., A 5-bp insertion in Mip causes recessive congenital cataract in KFRS4/Kyo rats, PLoS One 7 (2012) e50737.

[15] T. Kuramoto, S. Nakanishi, K. Yamasaki, K. Kumafuji, Y. Sakakibara, Y. Neoda, et al., Genetic quality control of the rat strains at the National Bio Resource Project Rat, Interdisciplinary Bio Central 2 (2010) 1-8.

[16] C.S. Moniaga, G. Egawa, H. Kawasaki, M. Hara-Chikuma, T. Honda, H. Tanizaki, et al., Flaky tail mouse denotes human atopic dermatitis in the steady state and by topical application with Dermatophagoides pteronyssinus extract, Am J Pathol 176 (2010) 2385-2393.

[17] T. Kuramoto, M. Yokoe, R. Hashimoto, H. Hiai, T. Serikawa, A rat model of hypohidrotic ectodermal dysplasia carries a missense mutation in the Edaradd gene, BMC Genet 12 (2011) 91. 
[18] T. Kuramoto, M. Kuwamura, S. Tokuda, T. Izawa, Y. Nakane, K. Kitada, et al., A mutation in the gene encoding mitochondrial $\mathrm{Mg}^{2+}$ channel MRS2 results in demyelination in the rat, PLoS Genet 7 (2011) e1001262.

[19] J. Gupta, E. Grube, M.B. Ericksen, M.D. Stevenson, A.W. Lucky, A.P. Sheth, et al., Intrinsically defective skin barrier function in children with atopic dermatitis correlates with disease severity, J Allergy Clin Immunol 121 (2008) 725-730 e722.

[20] N. Novak, New insights into the mechanism and management of allergic diseases: atopic dermatitis, Allergy 64 (2009) 265-275.

[21] M.K. Kagi, H. Joller-Jemelka, B. Wuthrich, Correlation of eosinophils, eosinophil cationic protein and soluble interleukin-2 receptor with the clinical activity of atopic dermatitis, Dermatology 185 (1992) 88-92.

[22] C. Koga, K. Kabashima, N. Shiraishi, M. Kobayashi, Y. Tokura, Possible pathogenic role of Th17 cells for atopic dermatitis, J Invest Dermatol 128 (2008) 2625-2630.

[23] T. Kobayashi, M. Glatz, K. Horiuchi, H. Kawasaki, H. Akiyama, D.H. Kaplan, et al., Dysbiosis and Staphyloccus aureus colonization drives inflammation in atopic dermatitis, Immunity 42 (2015) 756-766. 
[24] M. Asakawa, T. Yoshioka, I. Hikita, T. Matsutani, T. Hirasawa, A. Arimura, et al., WBN/Kob-Ht rats spontaneously develop dermatitis under conventional conditions: another possible model for atopic dermatitis, Exp Anim 54 (2005) 461-465.

[25] C. Michels, P. Goyal, N. Nieuwenhuizen, F. Brombacher, Infection with Syphacia obvelata (pinworm) induces protective Th2 immune responses and influences ovalbumin-induced allergic reactions, Infect Immun 74 (2006) 5926-5932.

[26] M.I. Asher, S. Montefort, B. Bjorksten, C.K. Lai, D.P. Strachan, S.K. Weiland, et al., Worldwide time trends in the prevalence of symptoms of asthma, allergic rhinoconjunctivitis, and eczema in childhood: ISAAC Phases One and Three repeat multicountry cross-sectional surveys, Lancet 368 (2006) 733-743.

[27] I.A. Deckers, S. McLean, S. Linssen, M. Mommers, C.P. van Schayck, A. Sheikh, Investigating international time trends in the incidence and prevalence of atopic eczema 1990-2010: a systematic review of epidemiological studies, PLoS One 7 (2012) e39803.

[28] H. Kawasaki, K. Nagao, A. Kubo, T. Hata, A. Shimizu, H. Mizuno, et al., Altered 
stratum corneum barrier and enhanced percutaneous immune responses in filaggrin-null mice, J Allergy Clin Immunol 129 (2012) 1538-1546 e1536.

[29] S. Ansar Ahmed, W.J. Penhale, N. Talal, Sex hormones, immune responses, and autoimmune diseases. Mechanisms of sex hormone action, Am J Pathol 121 (1985) 531-551.

[30] L.M. Pennell, C.L. Galligan, E.N. Fish, Sex affects immunity, J Autoimmun 38 (2012) J282-291.

[31] K. Horimukai, K. Morita, M. Narita, M. Kondo, H. Kitazawa, M. Nozaki, et al., Application of moisturizer to neonates prevents development of atopic dermatitis, $\mathrm{J}$ Allergy Clin Immunol 134 (2015) 824-830 e826. 


\section{Figure legends}

Fig. 1. Spontaneous dermatitis in Kyoto Fancy Rat Stock 4 rats. (A) Clinical photographs of 5-, 6- and 7-month-old Kyoto Fancy Rat Stock 4 (KFRS4) rats. (B) Total skin lesion severity scores (TSLSS) for male and female KFRS4 rats. Females showed significantly higher scores than age-matched males after 6 months of age. (C) Scratching behavior of female KFRS4 rats. (D) Total epidermal water loss (TEWL) through the dorsal skin of 5- and 8-week-old female PVG and KFRS4 rats. *: $P<0.05$; **: $P<0.01$

Fig. 2. Histological observations of the skin lesions of KFRS4 rats. (A, B) HE-stained sections of the dermatitis region in 7-month-old KFRS4 rats. In the eczematous lesions $(A$; original magnification $\times 200$ ), epidermal hyperplasia with mild spongiosis and infiltration of lymphocytes in the epidermis and superficial dermis were observed. In the progressed lesion $(\mathrm{B}$; original magnification $\times 40$ ), severe infiltrations of lymphocytes and eosinophils (inset; original magnification $\times 400$ ) in the dermis and ulcers were observed. (C) TB-stained section of the dermatitis region of KFRS4 rats 
(left, original $\times 400$ ) and the number of mast cells per field under 400-fold magnification

(right). (D) Immunohistochemical staining of CD4 and CD8 in the skin of 6-month-old

KFRS4 rats. Both $\mathrm{CD}^{+}$and $\mathrm{CD}^{+}$lymphocytes (arrows) were observed in the skin lesions but not in the normal skin of KFRS4 rats (left, original magnification $\times 400$ ). The number of $\mathrm{CD}^{+}$and $\mathrm{CD}^{+}$cells per field under 400-fold magnification (right). ${ }^{* *}: P<$ 0.01 .

Fig. 3. Immunological status of KFRS4 rats. (A) Total plasma IgE levels in male (open) and female (closed) KFRS4 rats. Females showed significantly higher IgE levels than age-matched males. (B) Expression levels of cytokines in the lymph nodes (LNs) of the dermatitis-affected female KFRS4 rats. (C) Number of eosinophils in the peripheral blood of the dermatitis-affected and non-affected KFRS4 rats. *: $P<0.05$; $* *: P<0.01$.

Fig. 4. Effects of ointments on dermatitis in KFRS4 rats. (A) TSLSS of ointment-treated and non-treated KFRS4 rats (left) and clinical photographs of 7-month-old rats (right). Note that topical application of betamethasone ointment 
greatly suppressed the dermatitis. (B) Scratching behavior in ointment-treated and non-treated KFRS4 rats. (C) HE-stained sections of the skin on which ointment was applied (original magnification, $\times 40)$. All results are shown as means \pm SD $(\mathrm{n}=6) .{ }^{*} P<$ 0.05 . 


\section{Author contributions}

TK and TS designed the research. MY, DT, AY, AN, YH and KY performed the experiments. MT, MK and $\mathrm{HH}$ performed the pathological examination. TK, $\mathrm{HH}$ and MT wrote the paper. KK, HH and TS revised the manuscript. 
A

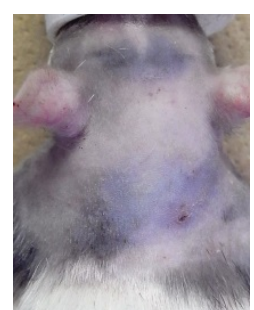

5 months

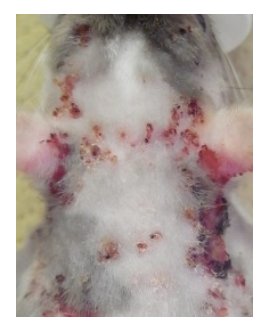

6 months
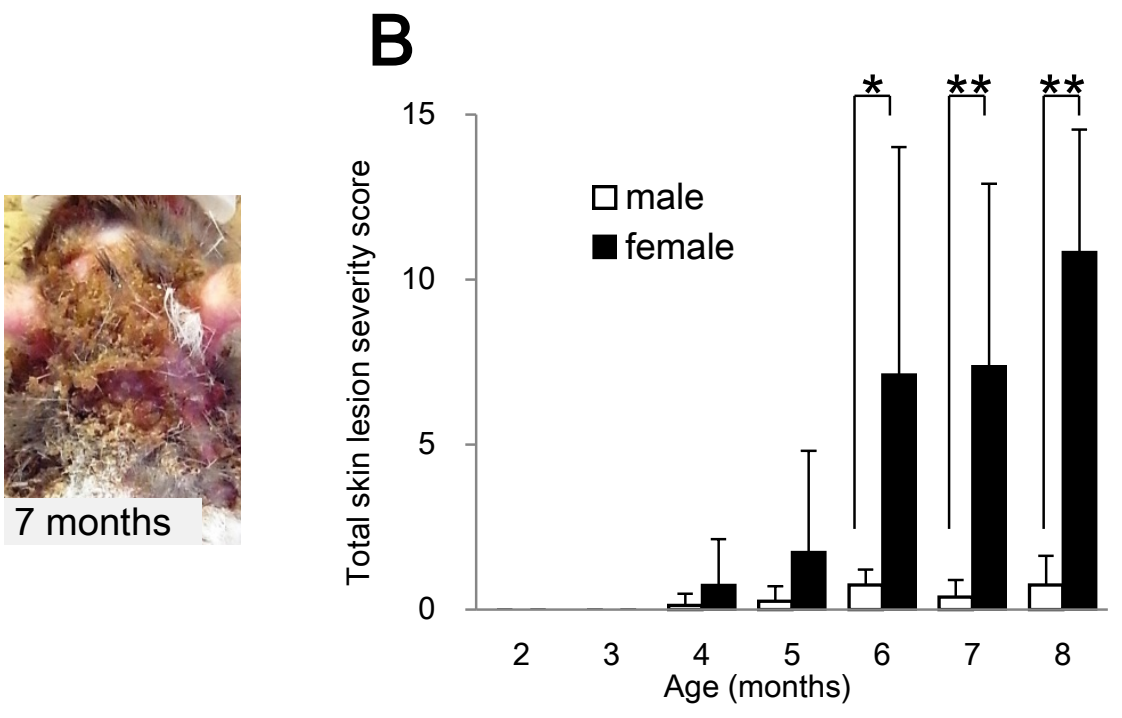

C

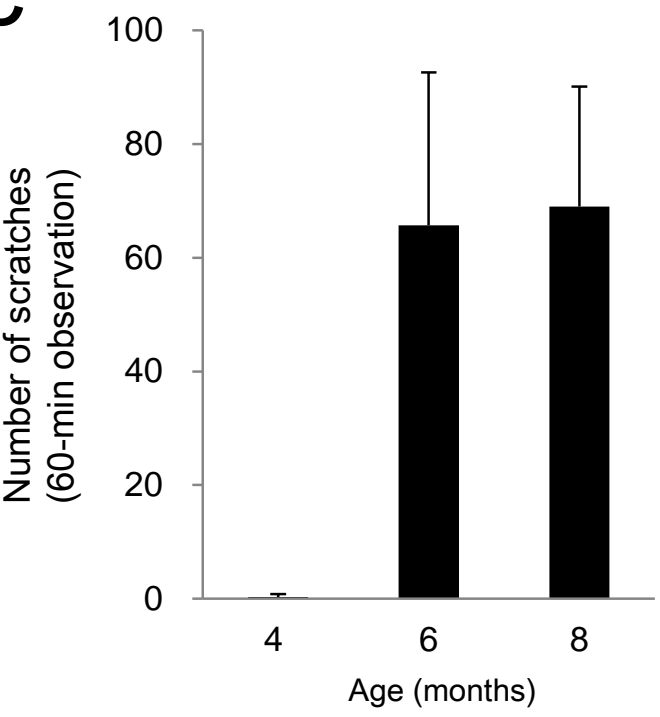

口PVG $-\mathrm{KFRS} 4$

D

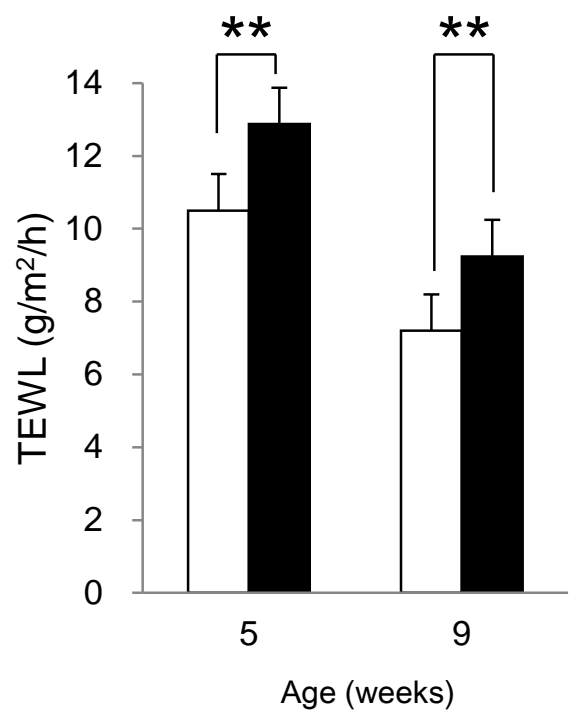


A
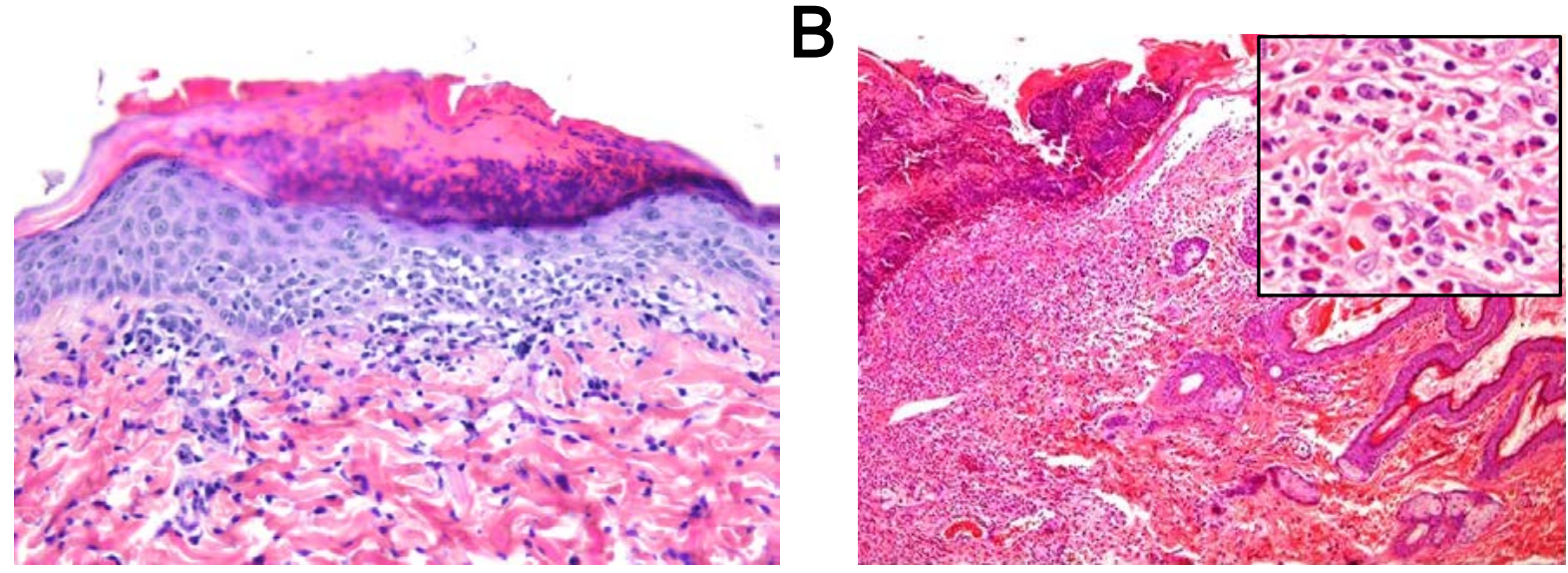

C
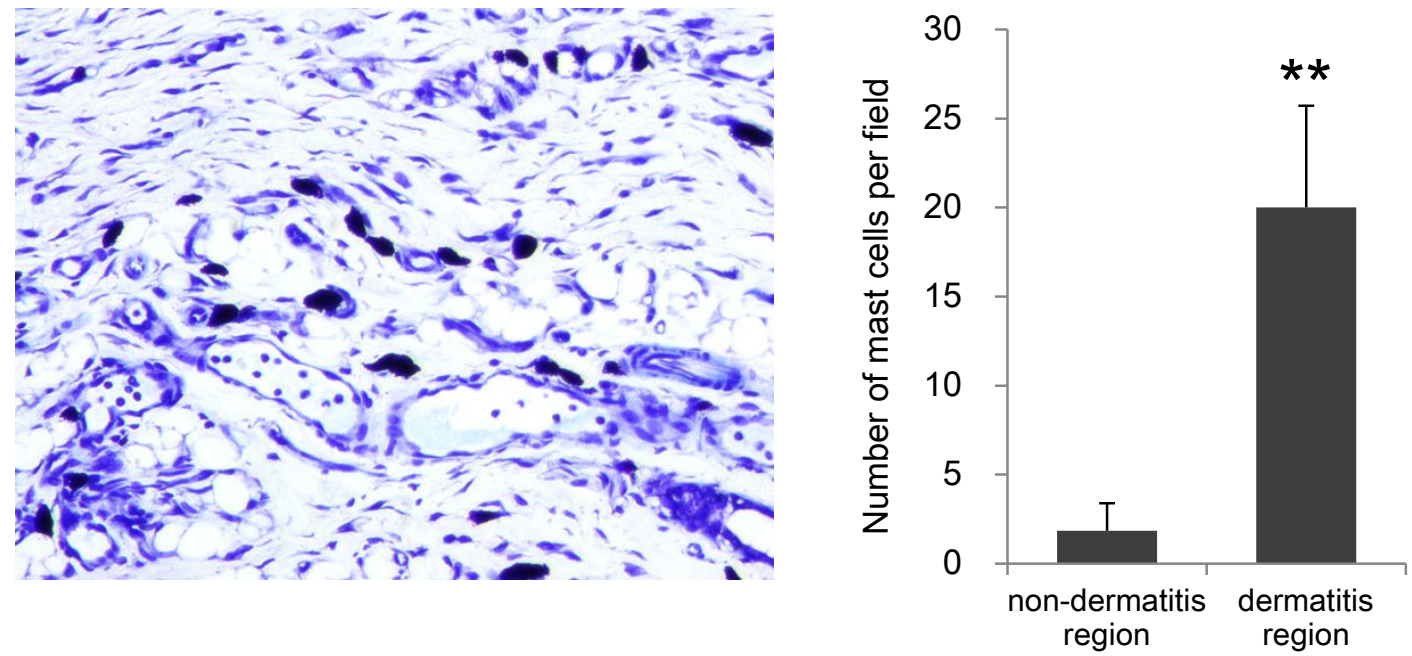

D

non-dermatitis region dermatitis region

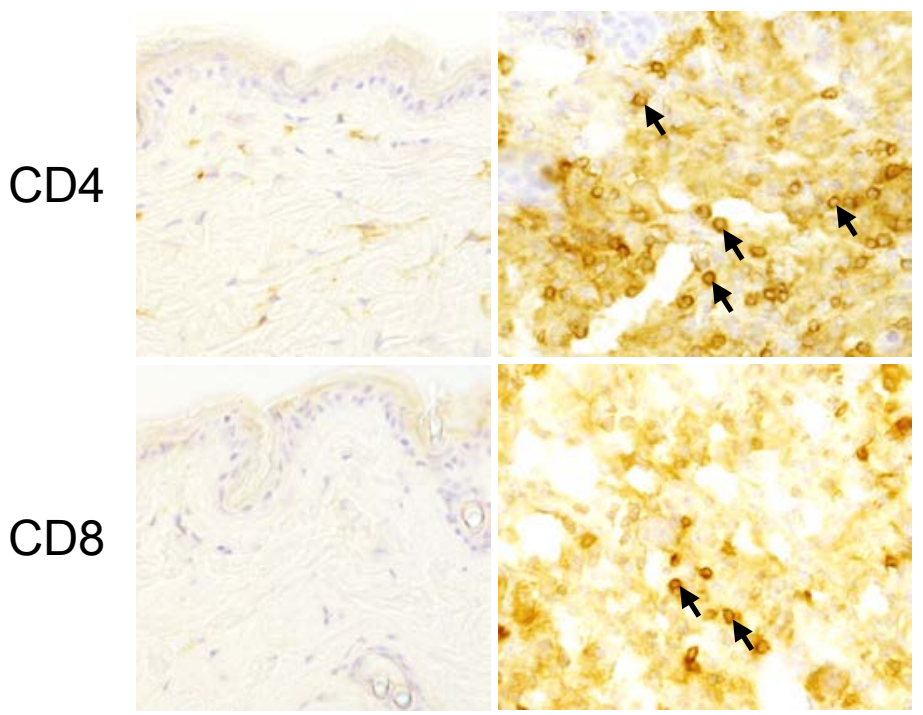

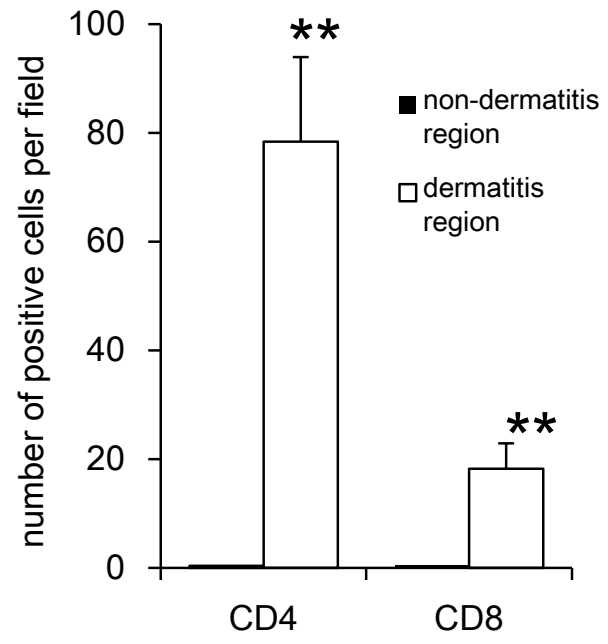


A

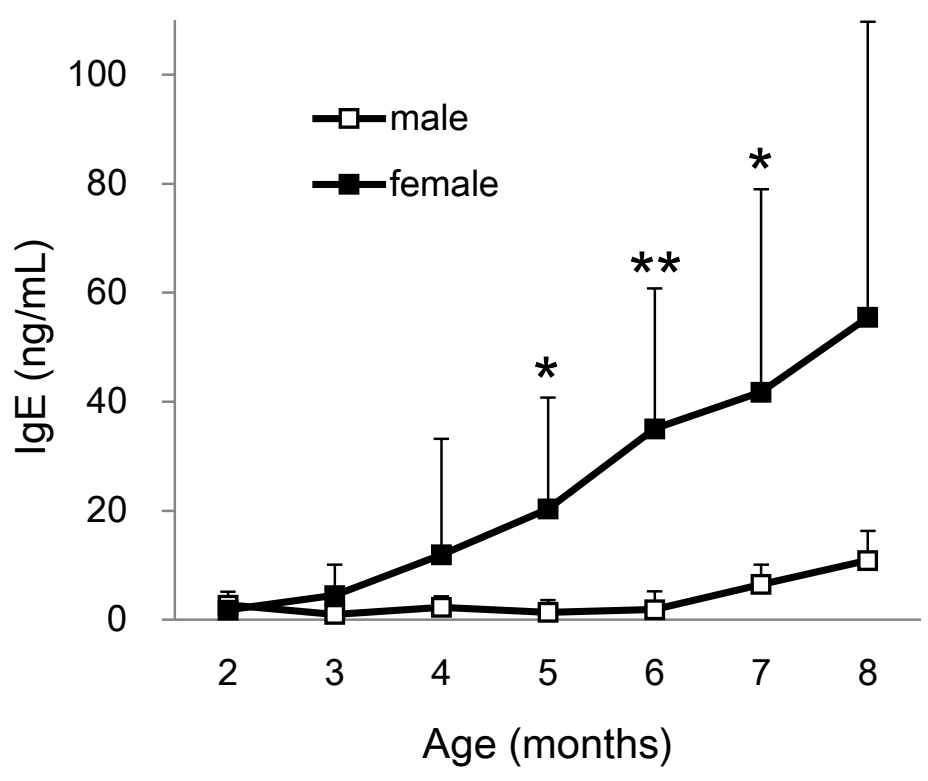

B

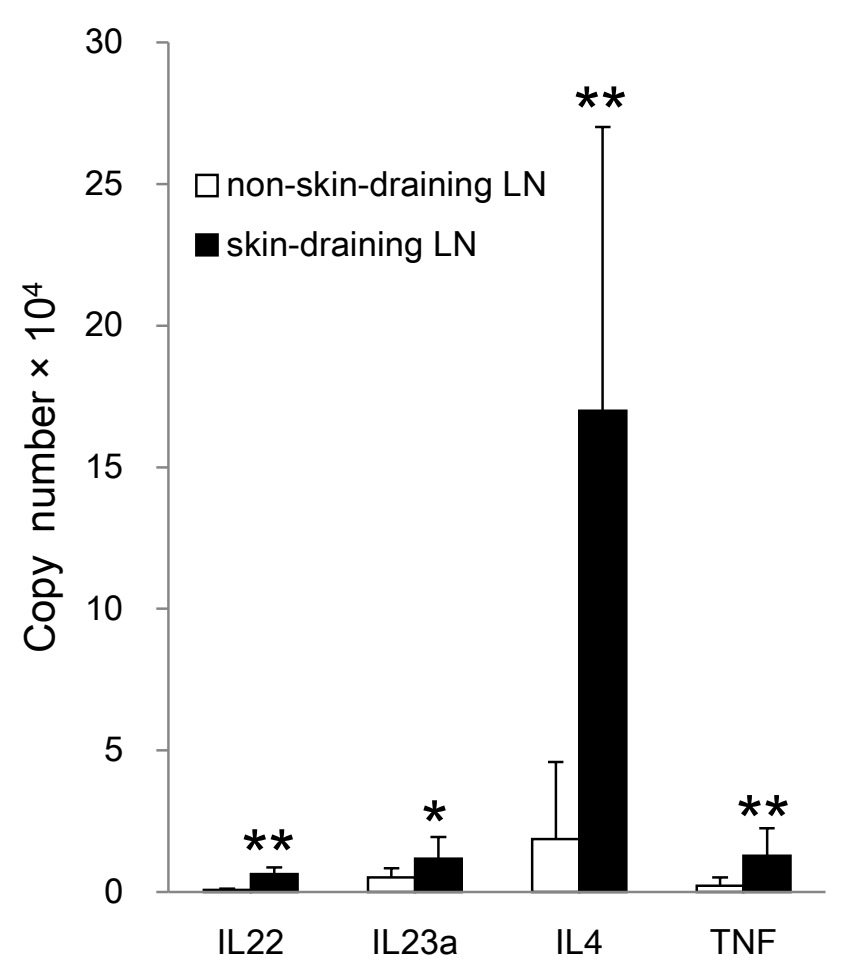

C

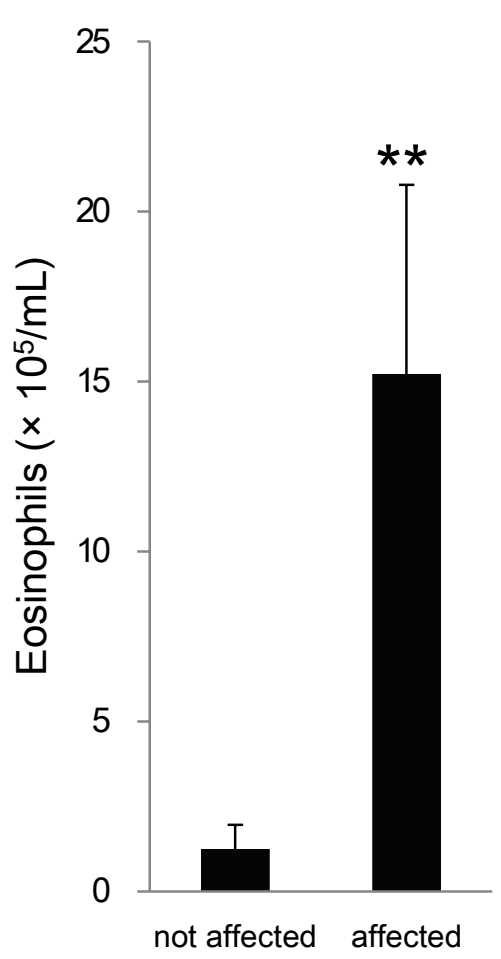

Figure 3 
A

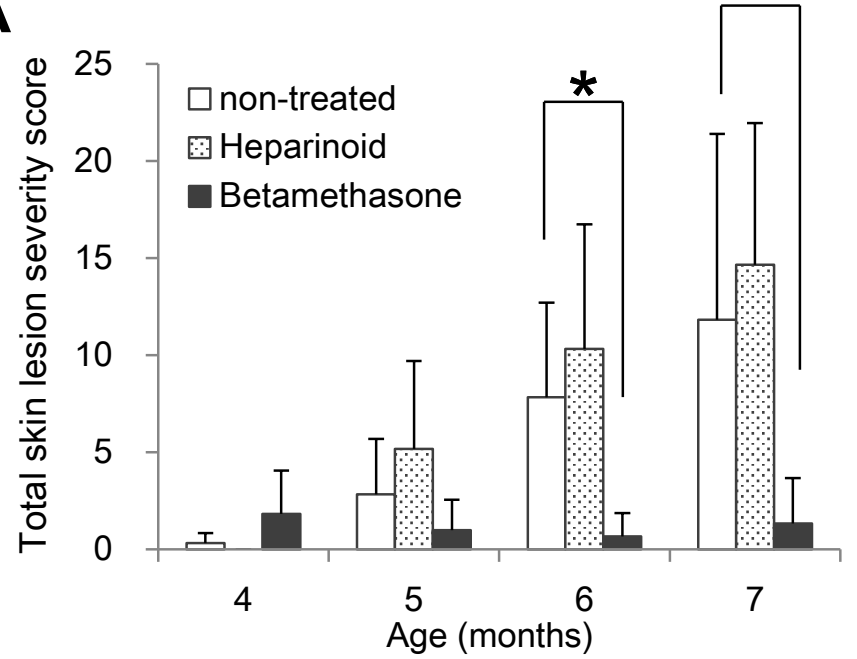

B

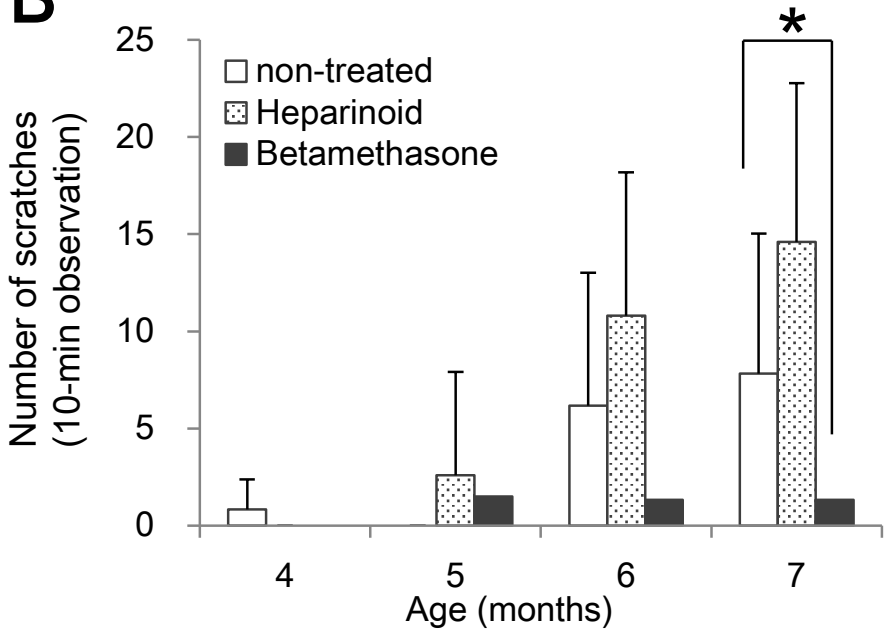

C
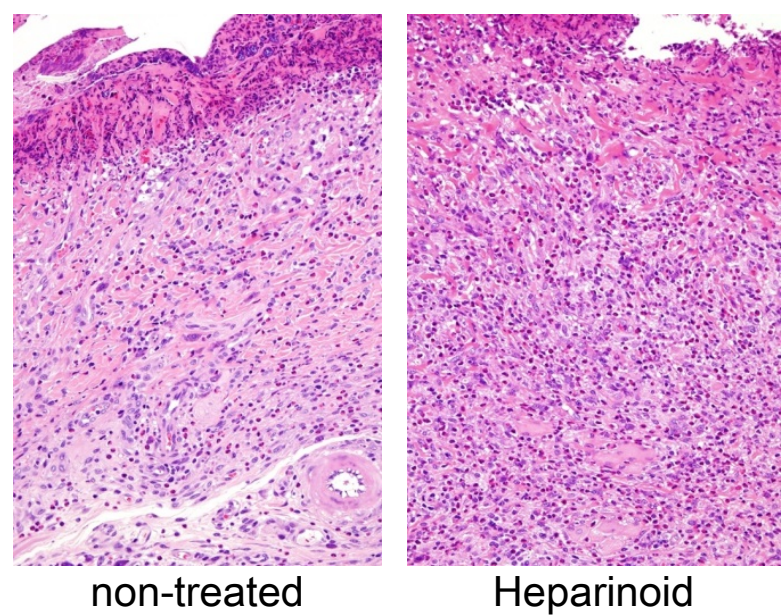

Age (months)
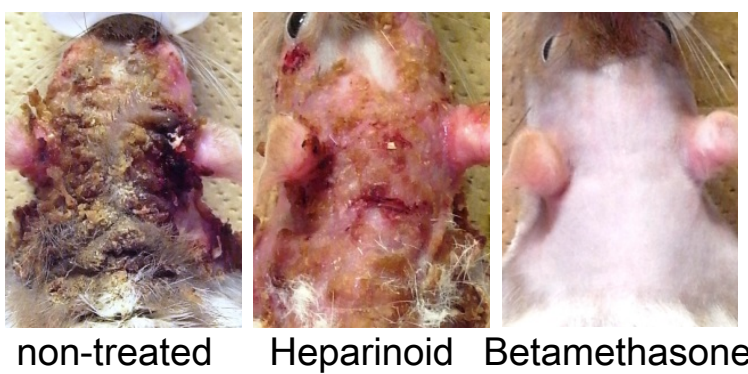

Heparinoid Betamethasone

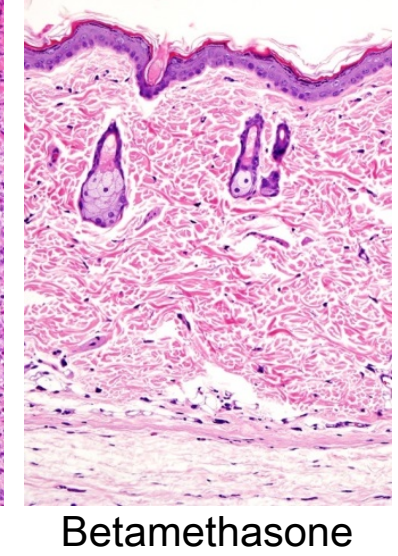


Supplementary Table 1 Primers used in the study

\begin{tabular}{|c|c|c|c|c|c|c|c|}
\hline \multirow{2}{*}{$\frac{\text { Symbol }}{\text { Ifng }}$} & \multirow{2}{*}{$\frac{\text { Gene name }}{\text { interferon gamma }}$} & \multirow{2}{*}{$\frac{\text { GenBank acc }}{\text { NM_138880.2 }}$} & \multirow{2}{*}{$\begin{array}{l}\text { Forward } \\
\text { AGGCCATCAGCAACAACATAAGTG }\end{array}$} & \multicolumn{2}{|c|}{ Forward start Reverse } & \multicolumn{2}{|c|}{ Reverse start Product size } \\
\hline & & & & 266 & GACAGCTTTGTGCTGGATCTGTG & 405 & 140 \\
\hline Il10 & interleukin 10 & NM_012854.2 & CAGACCCACATGCTCCGAGA & 110 & CAAGGCTTGGCAACCCAAGTA & 250 & 141 \\
\hline Il17a & interleukin 17A & NM_001106897.1 & 1 CTGATCAGGACGAGCGACCA & 40 & ACTGTAGCCTCCAGGTTCAGTAGCP & 126 & 87 \\
\hline Il22 & interleukin 22 & NM_001191988.1 & I GATGCTCTGCССАТСААСТСС & 94 & CCCGATGAGCCTGACATCTG & 228 & 135 \\
\hline Ill23a & Interleukin 23, alpha subunit p19 & NM_130410.2 & TCCCGGAATCTCTGCACACT & 416 & CAGACCTTGGCGGATCCTT & 601 & 186 \\
\hline Il4 & interleukin 4 & NM_201270.1 & TGCACCGAGATGTTTGTACCAGA & 169 & TTGCGAAGCACCCTGGAAG & 260 & 92 \\
\hline Ppia & peptidylprolyl isomerase A (cyclophilin & P NM_017101.1 & GGCAAATGCTGGACCAAACAC & 342 & AAACGCTCCATGGCTTCCAC & 476 & 135 \\
\hline Tnf & tumor necrosis factor & NM_012675.2 & AACTCGAGTGACAAGCCCGTAG & 400 & GTACCACCAGTTGGTTGTCTTTGA & 532 & 133 \\
\hline
\end{tabular}

